\title{
PREVALÊNCIA E FATORES ASSOCIADOS À VULNERABILIDADE EM IDOSOS: UMA REVISÃO
}

\author{
PREVALENCE AND FACTORS ASSOCIATED WITH VULNERABILITY IN ELDERLY: A \\ REVIEW
}

\section{Orlete Donato de Oliveira Miranda ${ }^{1} *$ Ezequiel Kleber Carpes Menezes $^{2} *$ Luiz Carlos Porcello $^{*}$ Marrone $^{3}$ * Maria Isabel Morgan Martins ${ }^{4}$}

\section{RESUMO}

O objetivo deste estudo foi identificar na literatura a prevalência de vulnerabilidade em idosos e os fatores associados. Trata-se de uma revisão de literatura, a busca foi realizada nas plataformas Portal CAPES, BVS Saúde e PUBMED, durante o período de 10 e 13 de março de 2020, após leitura chegouse a 11 publicações. Os resultados deste estudo demonstraram que a prevalência de vulnerabilidade oscilou de $32 \%$ entre idosos da comunidade até $89 \%$ entre os idosos hospitalizados. Sendo as principais variáveis associados à presença de vulnerabilidade: fatores sociodemográficos (idade, sexo, escolaridade), funcionais (incapacidade funcional), psicoemocionais (delirium, tristeza, desânimo, depressão, declínio cognitivo), autopercepção de saúde, doença cardíaca, polifarmácia e limitações sensoriais.

Palavras-chave: Vulnerabilidade; Idoso; Envelhecimento.

\section{ABSTRACT}

This research aimed to identify in the literature the prevalence of vulnerability in the elderly and the associated factors. This is a literature review of 11 publications, aiming to identify the prevalence of vulnerability in the elderly and associated factors. The search was carried out on the Portal CAPES, BVS Saúde and PUBMED platforms, during the period from March 10 to 13, 2020. The results of this study showed that the prevalence of vulnerability fluctuated from $32 \%$ among elderly people in the community, up to $89 \%$ among the hospitalized elderly. Being the main variables associated with the presence of vulnerability: sociodemographic factors (age, sex, education), functional (functional disability), psycho-emotional (delirium, sadness, discouragement, depression, cognitive decline), selfperceived health, heart disease, polypharmacy and limitations sensory.

Keywords: Vulnerability; Elderly; Aging.

\footnotetext{
${ }^{1}$ Graduada em Enfermagem pelo Centro Luterano de Ji-paraná. Especialista em Gestão Hospitalar / Universidade Paulista. E em Qualidade e Segurança no cuidado ao Paciente/ Instituto Sírio Libanês. Mestrado em Promoção da Saúde, Desenvolvimento Humano e Sociedade pela Universidade Luterana do Brasil, Brasil (2020). E-mail: orletedonato@ gmail.com.

${ }^{2}$ Graduado em Enfermagem pelo Centro Universitário São Lucas de Ji-Paraná, especialista em Docência em Enfermagem pela Faculdade de Venda Nova do Imigrante (FAVENI). E-mail: ezequielkleber@gmail.com

3 Graduado em medicina pela Universidade Luterana do Brasil (ULBRA), Doutor em Neurociências (Medicina) pela Pontificia Universidade Católica do Rio Grande do Sul. Professor de Neurologia da Faculdade de Medicina ULBRA e do Programa de Pós Graduação em Promoção da Saúde da ULBRA/Canoas. Pesquisador do Instituto do Cérebro do Rio Grande do Sul (PUCRS). Email: lcpmarrone@gmail.com

${ }^{4}$ Doutorado em Ciências Biológicas (Fisiologia) pela Universidade Federal do Rio Grande do Sul, Brasil (2003), Sócio do Conselho Regional de Biologia $3^{a}$ Região (PR, SC, RS), Brasil, Profa. Adjunta do Programa de Pós Graduação em Promoção da Saúde e Desenvolvimento Humano e Sociedade na Universidade Luterana do Brasil - ULBRA. E-mail: mimorganm@gmail.com
} 


\section{INTRODUÇÃO}

O envelhecimento é um processo marcado por uma diversidade de danos moleculares e celulares que causam uma perda gradual das reservas fisiológicas do organismo, resultando em uma elevação do risco de desordens e diminuição da capacidade intrínseca do organismo, que podem levar ao desfecho morte. No entanto, percebe-se que essas alterações somáticas são irregulares e nem sempre estão associadas à idade cronológica do indivíduo $^{(1)}$.

A população idosa brasileira vem seguindo padrões internacionais de crescimento. Em 2000, o número de idosos no Brasil encontrava-se em torno dos 14,2 milhões, tendo aumentado para 19,6 milhões em 2010, o que representava, naquele momento, cerca de $9,98 \%$ da população total. Este número continuará a crescer, podendo, em 2030, chegar a 41,5 milhões de idosos; para 2060, os dados são ainda mais alarmantes, chegando a aproximadamente 73,5 milhões de idosos no Brasil ${ }^{(2)}$.

Observando estes dados, é possível verificar que a população brasileira cresceu aceleradamente, se comparada a países europeus, o que resultou em uma insuficiência de programas de apoio e ações específicas voltadas para o atendimento das necessidades sociais, inclusive na área de saúde ${ }^{(3)}$. Nessa perspectiva, apesar de considerado um dos maiores avanços da humanidade, o fenômeno do envelhecimento populacional também é visto como um dos grandes desafios para saúde pública brasileira, uma vez que exige uma reorganização das questões sociais objetivando assistir às demandas específicas dessa população $^{(4)}$.

Os temas relacionados ao envelhecimento promoveram mudanças globais, estimuladas pela transição demográfica, ocasionando um novo modelo societário, fortemente caracterizado pela ascensão da expectativa de vida e envelhecimento da população ${ }^{(5)}$. No entanto, o processo de envelhecimento populacional tem refletido negativamente sobre as condições de vulnerabilidade, às quais os idosos encontramse suscetíveis. Dentre essas, é possível citar a presença de fragilidade física e o próprio contexto de vida individual $^{(6)}$.

A vulnerabilidade é definida como condição do indivíduo ou da coletividade que, por algum motivo, apresenta sua capacidade de autodeterminação reduzida, o que pode ocorrer em razão de déficit de poder, inteligência, educação, recursos, forças e outras causas que possam interferir na manutenção de seus 
interesses individuais ${ }^{(7)}$. A vulnerabilidade aplicada ao envelhecimento ainda tem seu uso bastante restrito, contudo, observa-se um crescente aumento do número de pesquisas ${ }^{(8)} \mathrm{e}$ profissionais do mundo acadêmico empenhados em realizar intervenções e identificar idosos sujeitos a danos ao bem-estar e à saúde ${ }^{(9)}$.

Uma proposta de avaliação da vulnerabilidade foi sugerida pelo autor ${ }^{(10)}$, por meio de um instrumento que avalia vulnerabilidade para morte e declínio funcional em idosos na comunidade. Desde então, este instrumento vem sendo utilizado em outros contextos, como preditor de mortalidade em pacientes oncológicos, cardiopatas e hospitalizados.

Desta forma, aprofundar-se nas questões relacionadas à vulnerabilidade em idosos pode ser considerada uma estratégia em saúde pública de grande importância, pois compreender o contexto associado aos agravos à saúde da pessoa idosa possibilitará sugestões

\section{MÉTODO}

Trata-se de revisão de literatura para a identificação de produções acerca do tema vulnerabilidade em idosos, prevalência e fatores associados. Para tanto, foi adotada a revisão integrativa da literatura para a de políticas públicas voltadas para criação de estratégias específicas, individuais e coletivas, para promoção, prevenção e recuperação da saúde do idoso. Tais estratégias podem ainda apresentar reflexos diretos sobre os determinantes de saúde dessa população, promovendo melhoria da qualidade de vida do idoso, família e coletividade, como também influenciar direta e indiretamente na redução de custos em saúde pública, decorrentes da recuperação da saúde por danos evitáveis.

Neste contexto, levando em consideração a temática envelhecimento, vulnerabilidade e sua importância para saúde pública, o presente estudo foi fundamentado sob a seguinte pergunta norteadora: qual a prevalência e fatores associados à vulnerabilidade em idosos?

Portanto, o objetivo desta pesquisa foi identificar na literatura a prevalência de vulnerabilidade em idosos e os fatores associados.

operacionalização do presente estudo, as seguintes etapas de construção foram respeitadas: 1) definição da pergunta de pesquisa; 2) definição dos critérios de inclusão de estudos e seleção da amostra; 3) organização 
dos estudos selecionados de forma crítica dos resultados de cada estudo; 5) organização das ideias centrais e definiçãa das temáticas a serem abordadas; 6) discussão.

A seleção dos estudos foi realizada por meio de buscas por publicações nas plataformas: Portal CAPES, BVS Saúde e PUBMED, durante o período de 10 e 13 de março de 2020. Foram adotados os seguintes critérios para seleção das publicações: artigos de texto completo, publicados entre 2014 a 2020, nos idiomas português, inglês e espanhol. Como critérios de exclusão HIV/AIDS, catástrofes naturais, fome e estressores ambientais, como ondas de calor. Foram utilizados os seguintes descritores da saúde (DeCS): "vulnerability study" AND "aged" AND "Eldery".

$\mathrm{Na}$ plataforma CAPES foram identificadas 292 publicações, das quais foram selecionadas 22 para leitura preliminar. $\mathrm{Na}$ plataforma BVSsalud, identificaram 91 publicações, das quais 20 foram selecionadas para leitura preliminar. $\mathrm{Na}$ plataforma PUBMED foram identificadas 408 publicações, das quais 14 compuseram leitura preliminar.

Para organização e tabulação dos dados, foi elaborado um instrumento de coleta de dados contendo: plataforma, periódico, ano de sistematizada em quadros e tabelas; 4) análise publicação, país do estudo, objetivo, método, conceito de vulnerabilidade, variáveis associadas e fragmentos do texto. Também foi elaborada uma planilha utilizando o software Microsoft Excel compilando os dados quantitativas.

Após a leitura de todos os 56 artigos, apenas 11 adequaram-se aos objetivos propostos e por conta disso foram selecionados para esta revisão. Então, uma nova leitura, feita de forma minuciosa, seguida da extração das variáveis desejáveis e organização da tabulação dos dados, precedeu à análise integrada dos principais resultados e reflexões acerca do tema, os quais compõem a presente discussão.

\section{RESULTADOS}

Levando em consideração os critérios de inclusão e exclusão das buscas, 11 publicações adequaram-se aos objetivos propostos e compuseram este estudo. Foi possível observar que, conforme apresentado no quadro abaixo (quadro 1), nos anos de 2015 e 2018, foram identificadas 4 (30\%) publicações; em 2016, 2 (15\%) publicações, enquanto que nos anos de 2014, 2017 e 2020 ocorreu apenas 1 (8\%) publicação em cada ano. 
Figura 1. Fluxograma da seleção amostral dos estudos incluídos na revisão sistemática. Ji-Paraná - RO, Brasil, 2020

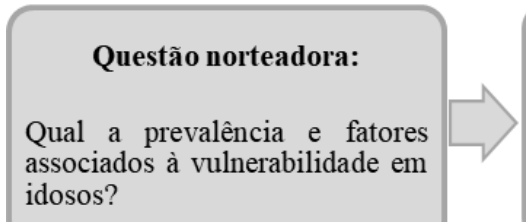

Busca em 10 e 13 de março de 2020

Descritores: "vulnerability study" AND "aged" AND "Eldery".

\section{Critérios de inclusão:}

Publicações nos idiomas português, inglês e espanhol, no período de 2014 a 2020, nas bases Portal CAPES, BVS Saúde e PUBMED
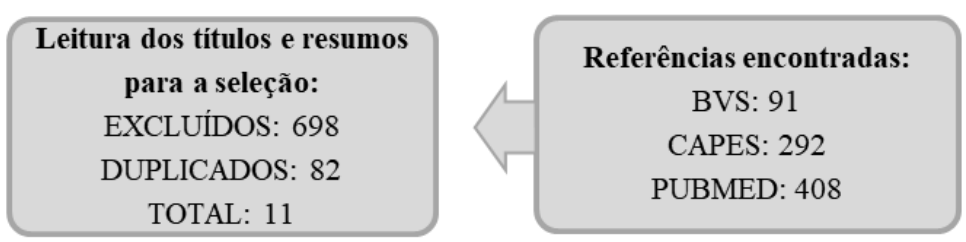

Fonte: elaboração própria, 2020.

Em relação aos países de origem dos estudos, constatou-se que o continente europeu foi o líder em estudos relacionados ao tema, com 5 (38\%) publicações, seguido pela América do Norte, com $3(23 \%)$ e América do Sul, com 3 (23\%) estudos. Oceania e Ásia computaram 1 (8\%) estudo cada. A maior parte dos estudos 11 (85\%) foram publicados em revistas internacionais e apenas $2(15 \%) \mathrm{em}$ periódicos nacionais.
Em relação ao delineamento dos estudos, $11(81 \%)$ foram quantitativos, sendo 5 (38\%) transversais, $5(38 \%)$ prospectivos e 1 $(8 \%)$ retrospectivo; e $2(15 \%)$ foram qualitativos exploratórios, ambos de revisão de literatura. As publicações estão apresentadas no quadro abaixo, de acordo com ano/autor, periódico, número da amostra e idade, local do estudo, tipo de estudo, instrumentos utilizados e descrição das variáveis associadas à vulnerabilidade.

Quadro 1: Relação das publicações relacionadas à prevalência de vulnerabilidade em idosos e fatores associados.

\begin{tabular}{|l|l|l|l|l|l|l|}
\hline $\begin{array}{l}\text { Autor/an } \\
\text { o }\end{array}$ & Periódico & $\begin{array}{l}\text { Amostra/id } \\
\text { ade }\end{array}$ & $\begin{array}{l}\text { Local do } \\
\text { estudo }\end{array}$ & Tipo & Instrumentos & $\begin{array}{l}\text { Variáveis } \\
\text { associadas à } \\
\text { vulnerabilidad } \\
\text { e }\end{array}$ \\
\hline (Ribeiro & Rev Bras & $\mathrm{N}: \quad 311$ & Belo & Quantitativo/ & IVCF - índice de & Autopercepçã \\
\hline
\end{tabular}




\begin{tabular}{|c|c|c|c|c|c|c|}
\hline $\begin{array}{l}\text { et al., } \\
\text { 2018) }\end{array}$ & $\begin{array}{l}\text { Enferm } \\
\text { [Internet] }\end{array}$ & $\begin{array}{l}\text { pacientes, } \\
\text { com idade } \\
\text { acima de } \\
60 \text { anos. } \\
\text { Sexo } \\
\text { feminino: } \\
216\end{array}$ & $\begin{array}{l}\text { Horizonte } \\
- \\
\text { MG/Brasi } \\
1\end{array}$ & $\begin{array}{l}\text { analítico/ } \\
\text { retrospectivo }\end{array}$ & $\begin{array}{l}\text { vulnerabilidade } \\
\text { clínico-funcional. }\end{array}$ & $\begin{array}{l}\text { o de saúde } \\
\text { tristeza } \\
\text { desânimo. }\end{array}$ \\
\hline $\begin{array}{l}\text { (Drewes } \\
\text { et al., } \\
2014)\end{array}$ & $\begin{array}{l}\text { PLOS } \\
\text { ONE }\end{array}$ & $\begin{array}{l}\mathrm{N}: \quad 2828 \\
\text { idosos com } \\
\text { idade acima } \\
\text { de } 75 \text { anos }\end{array}$ & Holanda & $\begin{array}{l}\text { Quantitativo/ } \\
\text { transversal/ } \\
\text { analítico }\end{array}$ & $\begin{array}{l}\text { Groningen Activity } \\
\text { Restriction Scale } \\
\text { (GARS); Mini-Mental } \\
\text { State Examination } \\
\text { (MMSE); } \\
\text { Instrumental activities } \\
\text { in daily living (IADL) } \\
\text { Basic Activities in } \\
\text { Daily Living (BADL); } \\
\text { Jong-Gierveld } \\
\text { Loneliness Scale } \\
\text { (DJG) }\end{array}$ & $\begin{array}{l}\text { Incapacidade } \\
\text { funcional, } \\
\text { número de } \\
\text { doenças } \\
\text { autorreferidas, } \\
\text { polifarmácia, } \\
\text { estado mental, } \\
\text { depressão. }\end{array}$ \\
\hline $\begin{array}{l}\text { (Barbosa } \\
\text { et al., } \\
2017)\end{array}$ & $\begin{array}{l}\text { Online } \\
\text { Brazilian } \\
\text { Journal of } \\
\text { Nursing }\end{array}$ & $\begin{array}{l}\mathrm{N}: \quad 368, \\
\text { idosos com } \\
60 \text { anos ou } \\
\text { mais; sexo } \\
\text { feminino } \\
253\end{array}$ & $\begin{array}{l}\text { João } \\
\text { Pessoa- } \\
\text { PB/Brasil } \\
.\end{array}$ & $\begin{array}{l}\text { Quantitativo/ } \\
\text { transversal/ } \\
\text { analítico }\end{array}$ & $\begin{array}{l}\text { VES-13; índice de } \\
\text { vulnerabilidade social }\end{array}$ & $\begin{array}{l}\text { Percepção de } \\
\text { saúde ruim, } \\
\text { ou regular, } \\
\text { dificuldades } \\
\text { no } \\
\text { desempenho } \\
\text { de atividades } \\
\text { de vida diária. }\end{array}$ \\
\hline $\begin{array}{l}\text { (Beddoes } \\
\text {-Ley et } \\
\text { al., 2016) }\end{array}$ & $\begin{array}{l}\text { BMC } \\
\text { Geriatrics }\end{array}$ & $\begin{array}{l}\mathrm{N}: \quad 1380 \\
\text { idoso com } \\
70 \text { anos ou } \\
\text { mais, sexo } \\
\text { feminino } \\
746\end{array}$ & $\begin{array}{l}\text { Victória/ } \\
\text { Austrália }\end{array}$ & $\begin{array}{l}\text { Quantitativo/ } \\
\text { transversal/ } \\
\text { analítico }\end{array}$ & VES-13 & $\begin{array}{l}\text { A redução da } \\
\text { força, redução } \\
\text { da mobilidade } \\
\mathrm{e} \\
\text { incapacidade } \\
\text { funcional. }\end{array}$ \\
\hline $\begin{array}{l}\text { (Kroc et } \\
\text { al., 2016) }\end{array}$ & $\begin{array}{l}\text { European } \\
\text { Geriatric } \\
\text { Medicine }\end{array}$ & $\begin{array}{l}\text { N: } 864 \\
\text { idosos; } \\
\text { acima de } \\
60 \text { anos; } \\
\text { sexo } \\
\text { feminino } \\
615\end{array}$ & $\begin{array}{l}\text { Lodz/Pol } \\
\text { ônia. }\end{array}$ & $\begin{array}{l}\text { Quantitativo/ } \\
\text { transversal/ } \\
\text { analítico }\end{array}$ & $\begin{array}{l}\text { VES-13, ADL, IADL, } \\
\text { MMSE e GDS }\end{array}$ & $\begin{array}{l}\text { ADL, IADL, } \\
\text { MMSE. }\end{array}$ \\
\hline $\begin{array}{l}\text { (Wang et } \\
\text { al., 2018) }\end{array}$ & $\begin{array}{l}\text { Archives } \\
\text { of } \\
\text { Gerontolo } \\
\text { gy and } \\
\text { Geriatrics }\end{array}$ & $\begin{array}{lr}\text { N: } & 2184 \\
\text { com } & 65 \\
\text { anos } & \text { ou } \\
\text { mais } & \text { de } \\
\text { idade. } & 1192 \\
(54.58 \%) \\
\text { dor sexo } \\
\text { masculino. }\end{array}$ & $\begin{array}{l}\text { Taiwan/C } \\
\text { hina. }\end{array}$ & $\begin{array}{l}\text { Quantitativo/ } \\
\text { longitudinal } \\
\text { prospectivo }\end{array}$ & 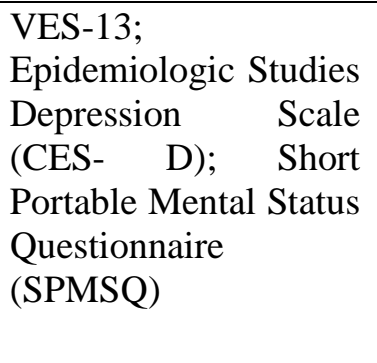 & $\begin{array}{l}\text { Sexo } \\
\text { masculino, } \\
\text { estado civil, } \\
\text { limitações } \\
\text { físicas e } \\
\text { auditiva. }\end{array}$ \\
\hline
\end{tabular}




\begin{tabular}{|c|c|c|c|c|c|c|}
\hline $\begin{array}{l}\text { (Bell et } \\
\text { al., 2015) }\end{array}$ & $\begin{array}{l}\text { BMJ } \\
\text { Open }\end{array}$ & $\begin{array}{lr}\mathrm{N}: & 445 ; \\
\text { acima } & \text { de } \\
65 & \text { anos; } \\
318 \text { sexo } \\
\text { feminino. }\end{array}$ & $\begin{array}{l}\text { Tennesse } \\
\text { e /USA }\end{array}$ & $\begin{array}{l}\text { Quantitativo/ } \\
\text { prospectivo/ } \\
\text { observaciona } \\
1\end{array}$ & $\begin{array}{lr}\text { VES-13; } & \text { CES-D; } \\
\text { SPMSQ; } & \text { s-TOFHLA; } \\
\text { SNS; } & \text { PHQ-8; } \\
\text { ENRICHD; } & \text { ESSI; } \\
\text { HRS; MIDUS. } & \end{array}$ & $\begin{array}{l}\text { Consultas } \\
\text { prévias, } \\
\text { hospitalização } \\
\text { e } \\
\text { atendimentos } \\
\text { de } \\
\text { emergência, } \\
\text { diagnóstico de } \\
\text { doença } \\
\text { cardíaca } \\
\text { depressão. }\end{array}$ \\
\hline $\begin{array}{l}\text { (Jeffery } \\
\text { et al., } \\
2018)\end{array}$ & $\begin{array}{l}\text { Archives } \\
\text { of } \\
\text { Gerontolo } \\
\text { gy and } \\
\text { Geriatrics }\end{array}$ & $\begin{array}{lr}\mathrm{N} & 188 ; \\
\text { acima } & \mathrm{de} \\
65 \text { anos; } \\
102 \text { sexo } \\
\text { feminino }\end{array}$ & $\begin{array}{l}\text { Nashville, } \\
\text { Tennesse } \\
\text { e, United } \\
\text { States }\end{array}$ & $\begin{array}{l}\text { Quantitativo/ } \\
\text { longitudinal } \\
\text { prospectivo/ } \\
\text { analítico }\end{array}$ & $\begin{array}{lr}\begin{array}{l}\text { Barthel; Life } \\
\text { Assessment }\end{array} & \begin{array}{l}\text { Space } \\
\text { (LSA), } \\
\text { VES-13, }\end{array} \\
\text { Dementia } & \text { AD8 } \\
\text { Injury } & \text { Screen, } \\
\text { Comorbidity Index 3, } & \text { Inderity } \\
\text { Cognitive Decline in } \\
\text { the Elderly (IQCDE) }\end{array}$ & $\begin{array}{l}\text { Declínio } \\
\text { cognitivo, } \\
\text { comorbidades. }\end{array}$ \\
\hline $\begin{array}{l}\text { (Deckx et } \\
\text { al., 2015) }\end{array}$ & $\begin{array}{l}\text { BMC } \\
\text { Family } \\
\text { Practice }\end{array}$ & $\begin{array}{lr}\text { N: } & 503 ; \\
\text { acima } & 70 \\
\text { anos; } & 244 \\
\text { sexo } & \\
\text { feminino } \\
\text { (106 com } \\
\text { diagnóstico } \\
\text { de câncer); } \\
110 \text { sexo } \\
\text { masculino } \\
\text { (28 com } \\
\text { diagnóstico } \\
\text { de câncer) }\end{array}$ & Holanda & $\begin{array}{l}\text { Quantitativo/ } \\
\text { longitudinal/ } \\
\text { prospectivo }\end{array}$ & 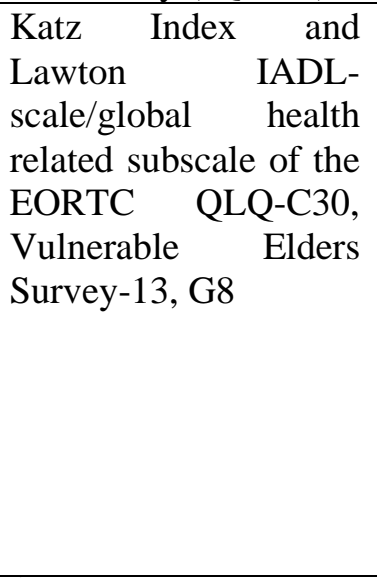 & $\begin{array}{l}\text { Escores } \\
\text { elevados nas } \\
\text { escalas } \\
\text { estavam } \\
\text { associadas a } \\
\text { um maior } \\
\text { risco de } \\
\text { declínio } \\
\text { funcional }\end{array}$ \\
\hline $\begin{array}{l}\text { (Cardona } \\
\text { et al., } \\
2018 \mathrm{a})\end{array}$ & $\begin{array}{l}\text { Biomédic } \\
\text { a }\end{array}$ & $\begin{array}{l}\text { N: } 1514 \\
\text { idosos } \\
\text { acima de } \\
60 \text { anos, } \\
\text { sendo } 960 \\
(58,2 \%) \\
\text { mulheres. }\end{array}$ & Colômbia & $\begin{array}{l}\text { Quantitativo/ } \\
\text { transversal }\end{array}$ & $\begin{array}{l}\text { Índice de Kaiser- } \\
\text { Meyer-Olkin/ } \\
\text { MMSE }\end{array}$ & $\begin{array}{l}\text { Papel familiar } \\
\text { Sexo; } \\
\text { escolaridade. }\end{array}$ \\
\hline $\begin{array}{l}\text { (Calf et } \\
\text { al., 2020) }\end{array}$ & $\begin{array}{l}\text { European } \\
\text { Journal of } \\
\text { Emergenc } \\
\text { y } \\
\text { Medicine } \\
\end{array}$ & $\begin{array}{l}\text { N: } 196 \\
\text { Acima de } \\
65 \quad \text { anos, } \\
\text { sendo } 95 \\
\text { mulheres. } \\
\end{array}$ & Holanda & $\begin{array}{l}\text { Quantitativo/ } \\
\text { prospectivo }\end{array}$ & $\begin{array}{l}\text { Mini avaliação } \\
\text { nutricional } \\
\text { VMS-screening } \\
\text { Katz }\end{array}$ & $\begin{array}{l}\text { Idade, estado } \\
\text { nutricional e } \\
\text { incapacidade } \\
\text { funcional. }\end{array}$ \\
\hline
\end{tabular}

Dados da pesquisa, 2020. 
Em relação aos instrumentos empregados na avaliação da vulnerabilidade, observa-se que, ao todo, 25 instrumentos foram identificados. Entre estes, quatro ferramentas específicas para mensuração da vulnerabilidade, IVCF - índice de vulnerabilidade clínico-funcional ${ }^{(4)} \quad$ o Vulnerable Elders Survey-13 $3^{(13,14,15,16,17,18,19,20)}$, o Índice de Kaiser-Meyer-Olkin ${ }^{(21)}$ e o VMSscreening ${ }^{(22)}$.

Além dos instrumentos de avaliação da vulnerabilidade, outros instrumentos inespecíficos também foram aplicados concomitantemente, como forma de validação dos dados, subsidiando a coleta ou compondo o quadro de variáveis nas respectivas análises. Dentre os instrumentos de avaliação do estado mental, destacaram-se os instrumentos: Short Portable Mental Status Questionnaire $(\mathrm{SPMSQ})^{(17,18)}$; Mini-Mental State Examination (MMSE) $^{(13,14,16,21)}$; AD8 Dementia Screen ${ }^{(19)}$; Cognitive Decline in the Elderly (IQCDE) ${ }^{(19)}$; Midlife Development in the United States (MIDUS) ${ }^{(18)}$; e Short Portable Mental Status Questionnaire (SPMSQ) ${ }^{(17,18)}$.

O domínio funcional também foi um dos componentes avaliados no estudo, destacando-se, neste contexto, as atividades básicas de vida diária (ABVD) e as atividades instrumentais de vida diária (AIVD) $)^{(13,16,20)}$, avaliadas por meio do índice de Barthel $^{(19)}$, Katz e Lawton e Brody ${ }^{(20,22)}$. Além desses, outros dois instrumentos relacionados ao domínio funcional - The Life Space Assessment (LSA) ${ }^{(19)}$ e Groningen Activity Restriction Scale (GARS) ${ }^{(13)}$ - foram utilizados nos estudos. Escalas de depressão foram utilizadas em três estudos, sendo estas Center for Epidemiologic Studies Depression Scale $(\mathrm{CES}-\mathrm{D})^{(17,18)}$, Patient Health Questionnaire $(\mathrm{PHQ}-8)^{(18)}$ e Geriatric Depression Scale $(\mathrm{GDS})^{(16)}$.

Qualidade de vida, satisfação social e contato familiar foram avaliados pelos instrumentos: Global health related subscale of the EORTC (QLQ-C30) ${ }^{(20)}$, ENRICHD Social Support Inventory $(E S S I)^{(18)}$ e Health and Retirement Survey $(\mathrm{HRS})^{(18)}$. A capacidade de interpretar informações relacionadas à saúde e a autopercepção relacionada à realização de tarefas matemáticas foram avaliadas em um estudo $^{(18)}$, por meio dos instrumentos Short Test of Functional Health Literacy in Adults (sTOFHLA) e Subjective Numeracy Scale (SNS).

$O$ Injury Severity Score (ISS) foi utilizado para avaliação da gravidade dos casos e o Charlson Comorbidity Index (CCI), utilizado como preditor de mortalidade ${ }^{(19)}$.

A prevalência de vulnerabilidade foi descrita em 7 (54\%) estudos ${ }^{(13-15,17,18)}$. E é 
possível observa-se que a prevalência de vulnerabilidade apresentou divergências de valores, uma vez que tais aferições ocorreram em tempo, faixas etárias, regiões e contextos distintos. O maior índice de vulnerabilidade foi observado em um estudo australiano (89\%), desenvolvido pelos autores ${ }^{(15)}$. Tal achado justifica-se pela faixa etária da amostra, idosos acima de 70 anos, e pelo local escolhido para realização do estudo: um hospital.

Um estudo brasileiro (52\%), desenvolvido em João Pessoa-PB ${ }^{(14)}$, e outro americano $(54 \%)^{(18)}$ apresentaram semelhança em relação à prevalência de vulnerabilidade. $\mathrm{O}$ estudo americano foi realizado com idosos portadores de doença cardíacas, atendidos em um centro de referência no tratamento de doenças do coração, enquanto o estudo brasileiro avaliou idosos residentes da comunidade e com faixa etária (60 anos) inferior aos descritos pela referência norte americana (65 anos). Em razão dos contextos associados à escolha da amostra e às questões regionais, sociais e culturais impactando em ambas realidades e, apesar da semelhança entre a prevalência de vulnerabilidade, os estudos não podem ser tomados por base para comparações ou generalizações simétricas. Os estudos com menor prevalência de vulnerabilidade $^{(13,17)}$ foram realizados com idosos residentes na comunidade em países desenvolvidos, Holanda (32\%) e China (35\%).

Com relação a distribuição das amostra descritas em cada um dos estudos desta revisão, notou-se que a Holanda ${ }^{(13)}$ é que obteve a maior amostra (2828), seguida pela pesquisa realizada na China ${ }^{(17)}$ com 2184, Colômbia ${ }^{(21)}$ com 1514, Austrália $^{(15)}$ com 1380, Polônia ${ }^{(16)}$ com 864, dois artigos realizados nos Estados Unidos da América $^{(18,20)}$ (USA) com 503 e 445 respectivamente, duas pesquisas realizadas no Brasil $^{(4,14)}$ (368 e 311 respectivamente), Holanda $^{(22)}$ (196) e mais uma nos USA ${ }^{(19)}$ com 188.

\section{DISCUSSÃO}

Após análise das publicações, foram levantadas as seguintes temáticas de discussão: fatores sociodemográficos, funcionais, psicoemocionais (delirium, tristeza, desânimo, depressão, declínio cognitivo), autopercepção de saúde e qualidade de vida, doença cardíaca, polifarmácia e limitações sensoriais.

Dentre as variáveis sociodemográficas, destaca-se o sexo feminino que foi o mais prevalente em todos os estudos analisados ${ }^{(4,13-}$ $20,22,23)$ e o com maior índice de vulnerabilidade nas análises transversais, contudo, observa-se, em estudos longitudinais, que o sexo masculino apresenta uma probabilidade maior para o 
desfecho morte. Conforme descrito em estudo asiático $^{(17)}$, homens apresentaram $147 \%$ mais risco de morte que mulheres. Além disso, o estado civil casado também apresentou redução de mortalidade em $5 \%$.

Já o grupo das variáveis associadas ao aspecto funcional foram as que apresentaram maior impacto sobre vulnerabilidade, sendo retratadas de diversas formas pelos estudos analisados: incapacidade funcional $^{(13,15)}$; dificuldades na realização de ABVD e $\operatorname{AIVD}^{(16,20)}$; redução da mobilidade e da força $^{(15)}$; e limitações físicas ${ }^{(17)}$.

Diversos estudos $^{(7,22,24,25)}$, realizados nos mais diferentes contextos, abordam a relação entre capacidade funcional e vulnerabilidade, sendo importante ressaltar que o declínio das capacidades funcionais pode levar à dependência funcional, incapacitando o idoso para realização das atividades de vida diária. É comum que grupos em vulnerabilidade se apresentem dependentes para as atividades realizadas fora do domicílio, o que contribui para o isolamento do idoso ${ }^{(5)}$.

Na variável Psicoemocional o delirium, disfunção cerebral aguda causada por vários insultos nocivos, permanece descrito na literatura como causa de vulnerabilidade e instabilidade clínica entre idosos. Sabe-se que, usualmente, a presença do delirium é mais frequente durante o período de hospitalização, porém pode ocorrer com menor frequência na comunidade, levando à necessidade de utilização de serviços de emergência, frequentemente associado à presença dos piores desfechos, como aumento do tempo de internação e elevadas taxas de mortalidade ${ }^{(11)}$.

A variável humor é uma função indispensável na preservação da autonomia e manutenção da capacidade funcional, pois está associada às funções mentais, como nível de consciência, sensopercepção e pensamento, bem como motivação necessária para atividades ou participação social do idoso. É importante destacar que o rebaixamento do humor ou baixa motivação varia desde a tristeza isolada até a depressão maior. No entanto, os sinais e sintomas muitas vezes são ignorados pelos profissionais de saúde, por julgarem serem decorrentes do processo de senilidade, o que leva, muitas vezes, ao transtorno depressivo ${ }^{(4)}$.

O transtorno depressivo é superior a um simples período de tristeza, de pessimismo, queda do humor e baixa autoestima por uma perda ou por uma mudança radical na vida. É uma alteração emocional que está associada a um alto risco de morbidade e mortalidade ${ }^{(4)}$. Tal questão foi evidenciada em pesquisa realizada em Taiwan, na qual foi observada uma mortalidade de $29,63 \%$ entre os pesquisados 
que encontravam-se em processo depressivo ${ }^{(17)}$. A partir desse fato, observa-se que a depressão, juntamente com outros condicionantes, é um fator intrinsecamente associado à vulnerabilidade e mesmo nas situações em que a depressão não venha a ser um fator de risco para vulnerabilidade, tardiamente os sintomas depressivos podem aparecer em decorrência desta $^{(18)}$. Contudo, em estudo realizado com 445 pacientes maiores de 65 anos, não ficou evidente a relação entre o aparecimento da depressão e a vulnerabilidade.

O Declínio Cognitivo (DC) pode ser resultante de alterações fisiológicas esperadas durante $\mathrm{o}$ processo de envelhecimento, entretanto, algumas vezes são maiores que o esperado. Idosos, com tal comprometimento cognitivo, podem demonstrar limitações que interferem desde a realização de atividades cotidianas, perda da autonomia, até a sobrecarga de cuidadores. Essas perdas funcionais e psicossociais podem ser o estágio de transição para as demências e a depressão, que irão prejudicar sobremaneira a vivência do idoso no ambiente familiar e social ${ }^{(26,27)}$.

Vários são os fatores determinantes na capacidade cognitiva, tais como as condições individuais, sociais e ambientais. A escolarização é um dos exemplos que demonstram que quanto maior tempo de escolaridade, maior a influência no desenvolvimento de habilidades cognitivas do cérebro, bem como na prevenção do declínio cognitivo. Outro destaque é o idoso que permanece economicamente ativo. Aqueles que continuam no mercado de trabalho são mais autônomos na resolução dos problemas, mantêm o convívio social e auxiliam na prevenção de incapacidades cognitivas ${ }^{(26,27,28)}$.

Por sua vez a autopercepção de saúde é um conceito que vem sendo utilizado em pesquisas epidemiológicas em saúde do idoso, pois além de ser um indicador do bem-estar físico, social e mental dessa população, verifica o impacto das doenças crônicas não transmissíveis nas condições gerais de saúde ${ }^{(4)}$. Além disso, a qualidade de vida também pode ser considerada um desses indicadores ${ }^{(21)}$, uma vez que a maior parte dos instrumentos de qualidade de vida avaliam a autopercepção de saúde, conforme evidenciado em estudo transversal ${ }^{(27)}$.

Estudos $^{(4,14)}$ ressaltam que a percepção da saúde ruim ou regular é mais prevalente entre os idosos vulneráveis, ou seja, à medida em que o grau de dependência aumenta, levando à fragilidade, maior é a chance de o idoso perceber seu estado de saúde como negativo. Dentre os fatores que podem interferir na percepção de saúde do idoso, podem ser 
citados fatores sóciodemográficos, tais como idade, sexo e estilo de vida; e as condições de saúde, como os problemas físicos, psíquicos, emocionais, dentre outros, que refletem de forma negativa no desempenho de funções e, consequentemente, na autopercepção de saúde.

Com relação a doenças cardiovasculares os Pesquisadores ${ }^{(29)}$, tentaram identificar relações importantes entre estados de saúde e fragilidade em adultos mais velhos. A partir daí, foi observado que os múltiplos estados de saúde, como doenças crônicas, incluindo diabetes, doenças pulmonares, depressão e doenças cardiovasculares, que geralmente coexistem em idosos frágeis, influenciam em sua vulnerabilidade biológica.

A doença cardiovascular, especialmente a insuficiência cardíaca congestiva, compartilha origens comuns com a fragilidade em idosos. E a natureza crônica e fisicamente limitante da síndrome da ICC pode levar à redução da atividade física e à piora do estado funcional, demonstrando uma associação entre a doença vascular e a vulnerabilidade em adultos mais velhos. E embora as associações entre estados de vulnerabilidade e doença não resultem em fragilidade, elas sugerem que um mecanismo biológico comum subjacente vincule algumas doenças e vulnerabilidade ${ }^{(12,18)}$.
Outro assunto importante é o consumo de medicamentos elevado entre idosos brasileiros. O Estudo ${ }^{30}$ identificou que 28,1\% idosos faziam uso de três a quatro medicamentos e que tal uso aumenta com a idade. Sabe-se que alguns fatores podem contribuir para a elevação no consumo de medicamentos, tais como a não adesão a tratamentos não farmacológicos, a automedicação, o uso incorreto dos medicamentos, o recebimento de prescrição de diferentes médicos e o inadequado entendimento das recomendações médicas ${ }^{(31)}$.

A polifarmácia está associada à vulnerabilidade. Segundo o autor ${ }^{(32)}$, o uso de polimedicação por idosos pode aumentar a ocorrência de efeitos adversos, diminuição da biodisponibilidade de vitaminas e minerais, déficits nutricionais importantes e dificuldade de adesão ao tratamento farmacológico correto. Além disso, existe a possibilidade de potencialização dos efeitos farmacológicos ou precipitação da fórmula, pela interação de algum medicamento, devido associação de mais fármacos utilizados no tratamento de muitas comorbidades frequentes em idosos ${ }^{(33)}$.

E por fim, as perdas sensoriais estão associadas à vulnerabilidade individual na população idosa. Identificou-se que a ocorrência de problemas, tais como os 
auditivos, visuais e vocais, associam-se estatisticamente de forma significativa com a presença de vulnerabilidade. Além disso, apresentam correlação direta com riscos mais altos de depressão, quedas, qualidade de vida relacionada à saúde, entre outros, o que resulta em um risco aumentado de vulnerabilidade, morbidade e mortalidade ${ }^{(14,17)}$. Pacientes com dificuldades auditivas e vocais tendem a ter ansiedade e a se isolarem socialmente, o que resulta em maiores índices de depressão, com consequente vulnerabilidade do idoso ${ }^{(34)}$.

\section{CONSIDERAÇÕES FINAIS}

Este estudo permitiu identificar a prevalência de vulnerabilidade em pesquisas ao longo do mundo, nos mais diferentes contextos, como também os fatores associados. Entre os fatores associados estão as condições socioeconômicas, estado funcional, alterações psicoemocionais, autopercepção de saúde, doença cardiovascular, polifarmácia, limitações sensoriais. Foi possível identificar diferentes instrumentos utilizados para mensurar vulnerabilidade em diferentes contextos. A prevalência de vulnerabilidade nos estudos analisados oscilou de 32 a $89 \%$. Acredita-se que esta ampla margem seja em função da diferença de contextos como grupo analisado, idade, localização geográfica, instrumento utilizado e outros.

As limitações deste estudo foram a dificuldade da padronização do conceito vulnerabilidade, e a diversidade de contextos da amostra, o que acaba dificultando análises mais elaboradas. Espera-se que estes dados possam servir de embasamento para profissionais de saúde no sentido de compreender o contexto envelhecimento humano, tendo em vista a implementação de estratégias em saúde, visando a promoção, prevenção e recuperação da saúde do idoso.

\section{REFERÊNCIAS}

1. Organização Mundial da Saúde -. Relatório Mundial de Envelhecimento e Saúde. Organização Pan-Americana de Saúde. Brasília; 2015.

2. Brasil Ministério da Saúde. Atenção à Saúde da Pessoa Idosa e Envelhecimento. Ministério da Saúde. 2010. 46 p. Available from: http://bvsms.saude.gov.br/bvs/publicacoe s/atencao_saude_pessoa_idosa_envelheci mento_v12.pdf

3. Silva HS da, Lima ÂMM de, Galhardoni R. Envelhecimento bem-sucedido e vulnerabilidade em saúde: Aproximações e perspectives. Interface Commun Heal Educ. 2010;14(35):867-77.

4. Ribeiro EG, Matozinhos FP, De Lima Guimarães G, Do Couto M, Souza 
Azevedo R, Yovana I, et al. Autopercepção de saúde e vulnerabilidade clínico-funcional de idosos de Belo Horizonte/Minas Gerais. Rev Bras Enferm [Internet]. 2018;71(2):914-21. Available from: http://dx.doi.org/10.1590/0034-71672017-0135

5. Dos Santos AA, Pavarini SCI. Funcionalidade de idosos com alterações cognitivas em diferentes contextos de vulnerabilidade social. ACTA Paul Enferm. 2011;24(4):520-6. doi: 10.5216/ree.v13i2.10170

6. Luzardo AR, Medeiros M, Paula Júnior NF de, Wolkers PCB, Lima LSB, Santos SMA dos. Queda de idosos: desvelando situações de vulnerabilidade. REME Rev Min Enferm. 2017;21. doi: $10.5935 / 1415-2762.20170035$

7. Alexandre $\mathrm{T}$ da $\mathrm{S}$, Corona $\mathrm{LP}$, Nunes DP, Santos JLF, Duarte YA de O, Lebrão ML. Disability in instrumental activities of daily living among older adults: Gender differences. Rev Saude Publica. 2014;48(3):379-89. doi: 10.1590/S0034-8910.2014048004754

8. Maia F de OM, Duarte YA de O, Secoli SR, Santos JLF, Lebrão ML. Adaptação transcultural do Vulnerable Elders Survey -13 (VES-13): contribuindo para a identificação de idosos vulneráveis. Rev Esc Enferm USP. 2012;13(46):11622. Available from: http://www.scielo.br/pdf/reeusp/v46nspe /17.pdf

9. Salmazo-Silva H, Lima-Silva TB, Barros TC de, Oliveira EM de, Ordonez TN, Carvalho G, et al. Vulnerabilidade na velhice: definição e intervenções no campo da Gerontologia. Rev Kairós Gerontol. 2012;15(6):97-116. Available from:

https://revistas.pucsp.br/index.php/kairos /article/view/17289/12829

10. Saliba D, Elliott M, Rubenstein LZ, Solomon DH, Young RT, Kamberg CJ, et al. The vulnerable elders survey: A tool for identifying vulnerable older people in the community. J Am Geriatr Soc. 2001;49(12):1691-9. doi: 10.1046/j.1532-5415.2001.49281.x

11. Bellelli G, Mazzola P, Morandi A. Delirium as a marker of vulnerability in the elderly. Geriatr Care. 2015;1(1):1-4. Available from: http://www.pagepressjournals.org/index. $\mathrm{php} / \mathrm{gc} / \mathrm{article} / \mathrm{view} / 5472$

12. Walston JD. Connecting Age-Related Biological Decline to Frailty and LateLife Vulnerability. Nutr Inst Work Ser. 2015;83(1):1-10. doi: 10.1159/000382052.Connecting

13. Drewes YM, Blom JW, Assendelft WJJ, Stijnen T, Den Elzen WPJ, Gussekloo J. Variability in vulnerability assessment of older people by individual general practitioners: A cross-sectional study. PLoS One. 2014;9(11):1-7. doi: 10.1371/journal.pone.0108666

14. Barbosa KTF, Costa KN de FM, Pontes $\mathrm{M}$ de L de F, Batista PS de S, Oliveira FMRL de, Fernandes $M$ das GM. Envelhecimento e vulnerabilidade individual: um panorama dos idosos vinculados à estratégia saúde da famíliA. Texto Context Enferm. 2017;26(2):1-10.

15. Beddoes-Ley L, Khaw D, Duke M, Botti 
M. A profile of four patterns of vulnerability to functional decline in older general medicine patients in Victoria, Australia: A cross sectional survey. BMC Geriatr. 2016;16(1):1-12. Available from: http://dx.doi.org/10.1186/s12877-0160323-1

16. Kroc, Socha K, Sołtysik BK, CieślakSkubel A, Piechocka-Wochniak E, Błaszczak R, et al. Validation of the Vulnerable Elders Survey-13 (VES-13) in hospitalized older patients. Eur Geriatr Med. 2016;7(5):449-53. doi: 10.1016/j.eurger.2016.03.008

17. Wang J, Lin W, Chang LH. The linear relationship between the Vulnerable Elders Survey-13 score and mortality in an Asian population of communitydwelling older persons. Arch Gerontol Geriatr. 2018;74(May 2017):32-8. Available from: https://doi.org/10.1016/j.archger.2017.09 .005

18. Bell SP, Schnelle J, Nwosu SK, Schildcrout J, Goggins K, Cawthon C, et al. Development of a multivariable model to predict Vulnerability in older American patients hospitalised with cardiovascular disease. BMJ Open. 2015;5(8):1-8. doi: 10.1136/bmjopen2015-008122

19. Jeffery AD, Dietrich MS, Maxwell CA. Predicting 1-year disability and mortality of injured older adults. Arch Gerontol Geriatr. 2018;75(December 2016):1916. doi: 10.1016/j.archger.2018.01.003

20. Deckx L, Van Den Akker M, Daniels L, De Jonge ET, Bulens P, Tjan-Heijnen $\mathrm{VC}$, et al. Geriatric screening tools are of limited value to predict decline in functional status and quality of life: Results of a cohort study Service organization, utilization, and delivery of care. BMC Fam Pract. 2015;16(1). doi: 10.1186/s12875-015-0241-X

21. Cardona D, Segura Á, Segura A, Muñoz D, Jaramillo D, Lizcano D, et al. índice de vulnerabilidad de los adultos mayores: Medellín, Barranquilla y Pasto. Biomedica. 2018;38:1-44. doi: 10.7705/biomedica.v38i0.3846

22. Calf AH, Lubbers S, Van Den Berg AA, Van Den Berg E, Jansen CJ, Van Munster BC, et al. Clinical impression for identification of vulnerable older patients in the emergency department. Eur J Emerg Med. 2020;137-41. doi: 10.1097/MEJ.0000000000000632

23. Vieira KLD, Gomes VL de O, Borba MR, Costa CF da S. Atendimento da população masculina em unidade básica saúde da família: motivos para a (não) procura. Esc Anna Nery. 2013;17(1):120-7. doi: 10.1590/s141481452013000100017

24. Moraes EN de, do Carmo JA, de Moraes FL, Azevedo RS, Machado CJ, Montilla DER. Clinical-Functional Vulnerability Index-20 (IVCF-20): Rapid recognition of frail older adults. Rev Saude Publica. 2016;50:1-9. doi: 10.1590/S15188787.2016050006963

25. Jesus ITM de, Orlandi AA dos S, Grazziano E da S, Zazzetta MS. Fragilidade de idosos em vulnerabilidade social. Acta Paul Enferm. 2017;30(6):614-20. Available from: http://www.scielo.br/scielo.php?script=s ci_arttext\&pid=S0103- 
$21002017000600614 \& \operatorname{lng}=p t \& \operatorname{lng}=p t$

26. Mendes PA, Reiners AAO, Azevedo RC de S, Gaspar ACM, Segri NJ. Declínio Cognitivo: Conhecimentos, Atitudes E Práticas Preventivas De Idosos Em Comunidade. Cogitare Enferm. 2018;23(3). doi: 10.5380/ce.v23i3.55372

27. Szerwieski LLD, Cortez DAG, Bennemann RM, Silva ES, Cortez LER, Narriman R, et al. Capacidade cognitiva, estilo e qualidade de vida dos idosos: estudo transversal. Online Brazilian J Nurs. 2018;16(3):298. doi: $10.17665 / 1676-4285.20175723$

28. Campos CA et al. Levantamento de vulnerabilidades funcionais em idosos: possibilidades de atuação para a atenção primária à saúde. Sinapse Múltipla. 2017;6(2):235-40.

29. Pereira, Alexandre Alves FSAB, Neri AL. Risco de morte em idosos baseado no fenótipo de fragilidade e no índice de fragilidade: um estudo de revisão. Rev Bras Geriatr e Gerontol. 2017;20(2):274-87. doi: 10.1590/198122562017020.160102

30. Santos VP et al. Perfil de saúde de idosos muito velhos em vulnerabilidade social na comunidade. Rev Cuid. 2018;9(3):2322-37.

31. Bento IC, Anne M, Souza N. Associação entre número de medicamentos consumidos e marcadores nutricionais entre idosos com doenças crônicas: Pesquisa Nacional de Saude (2013). Rev Bras Geriatr e Gerontol. 2019;22(1):110.
32. Cabral JF, Silva AMC da, Mattos IE, Neves Á de Q, Luz LL, Ferreira DB, et al. Vulnerabilidade e fatores associados em idosos atendidos pela Estratégia Saúde da Família. Cien Saude Colet. 2019;24(9):3227-36. doi: 10.1590/141381232018249.22962017

33. Campos CA, Gama CC, Carvalho JPSS, Neves JM, Carneiro MGC, Costa MC, et al. Levantamento de vulnerabilidades funcionais em idosos: possibilidades de atuação para a atenção primária à saúde. Sinapse Múltipla. 2017;6(9):235-40. doi: 10.1590/1413-81232018249.22962017

34. Chiossi JSC, Roque FP, de Goulart BNG, Chiari BM. Impacto das mudanças vocais e auditivas na qualidade de vida de idosos ativos. Cienc e Saude Coletiva. 2014;19(8):3335-42. doi: 10.1590/141381232014198.07642013

Submissão: 2020-07-26

Aprovado: 2021-03-31 